\title{
Locality in Free String Field Theory - II.
}

\author{
J. Dimock* \\ Dept. of Mathematics \\ SUNY at Buffalo \\ Buffalo, NY 14214
}

November 2, 2018

\begin{abstract}
We study the covariant free bosonic string field theory and explore its locality (causality) properties. We find covariant string fields which are strictly local and covariant, but act on an unconstrained Hilbert space with an indefinite inner product. From these we also define observable fields which act on the physical Hilbert space with an definite inner product. These are shown to be approximately local.
\end{abstract}

\section{Contents}

1 Introduction 2

1.1 Overview . . . . . . . . . . . . . . . . . . . . . . . . . . . . . . . . . . . . . .

1.2 Lorentz invariant measures . . . . . . . . . . . . . . . . . . . . . . . . 2

\begin{tabular}{|lll}
2 & The single string & 5
\end{tabular}

2.1 Pre-constrained . . . . . . . . . . . . . . . . . . . . 5

2.2 Reconfigured . . . . . . . . . . . . . . . . . . . . . . . 9

2.3 Constrained . . . . . . . . . . . . . . . . . . . . . . . . . . . 11

2.4 Physical States . . . . . . . . . . . . . . . . . . . . . 13

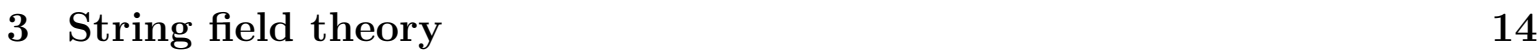

3.1 String field equation . . . . . . . . . . . . . . . . . . . . . . . . . 14

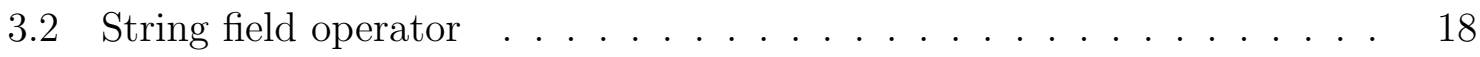

${ }^{*}$ Research supported by NSF Grant PHY0070905 


\section{Introduction}

\section{$1.1 \quad$ Overview}

We continue to explore the locality properties of free bosonic string field theory. The guiding question is whether one can find string fields which satisfy the field equation, are strictly local (causal), and are Lorentz covariant. Success in this quest would mean one could define an algebra of local observables of the type that one usually considers in quantum field theory [11]. We do not necessarily expect to succeed in this quest, but do expect that the ways in which we fall short will be of interest.

In an earlier work [16], [15], [5], the problem was considered in the light cone gauge and string fields were found which were local with respect to the center of mass coordinate. However these fields were not Lorentz covariant.

In the present paper we work with a Lorentz covariant formalism right from the start. It is the so-called "old covariant quantization" in which one quantizes first and then imposes the constraint. Before imposing constraints, we are able to construct string field operators which are Lorentz covariant and local in the sense that the commutator of two fields vanishes when the center of mass coordinates are spacelike separated. However the field operators act on a Hilbert space with an indefinite inner product. Once the constraints are imposed one obtains a definite inner product. On this space we also define covariant field operators called observable fields. For these observable fields we establish an approximate locality property.

Our results seem to be consistent with the treatment of Hata and Oda [12] who work in a BRST formalism. A general account of string field theory can be found in Thorn [20]

Another goal of this work is to solidify the mathematical foundations of covariant string theory. For earlier work in this direction see Grundling and Hurst [10].

\subsection{Lorentz invariant measures}

We start by developing some facts about Lorentz invariant measures on the mass shells. (See also [18]). For any real number $r$ let

$$
V_{r}=\left\{p \in \mathbb{R}^{d}-\{0\}: p^{2}+r=0\right\}
$$

Here $p^{2}=p \cdot p=\sum_{\mu \nu} \eta_{\mu \nu} p^{\mu} p^{\nu}=-\left(p^{0}\right)^{2}+|\vec{p}|^{2}$ is the Lorentz inner product. The Lorentz group is all nonsingular linear transformations preserving $p^{2}$ and it acts on $V_{r}$.

First we define a Lorentz invariant volume element on $V_{r}$. Let $\tau=d p^{0} \wedge d p^{1} \ldots \wedge d p^{d-1}$ be the volume element in $\mathbb{R}^{d}$. With $\alpha(p)=-p^{2}$ define $\sigma$ to be the unique $d-1$ form on $V_{r}$ such that

$$
d \alpha \wedge \sigma=\tau
$$


Transforming this by a proper Lorentz transformation $\Lambda$ we have $d(\alpha \circ \Lambda) \wedge \Lambda^{*} \sigma=\Lambda^{*} \tau$. But $\alpha \circ \Lambda=\alpha$ by definition and $\Lambda^{*} \tau=\tau$ since $\operatorname{det} \Lambda=1$. Thus $d \alpha \wedge \Lambda^{*} \sigma=\tau$ and hence $\Lambda^{*} \sigma=\sigma$.

Now $\int_{V_{r}} f \sigma$ is defined for continuous functions $f$ with compact support on $V_{r}$. The map $f \rightarrow \int_{V_{r}} f \sigma$ is positive and hence there is a positive measure $\mu_{r}$ on $V_{r}$ such that

$$
\int_{V_{r}} f d \mu_{r}=\int f \sigma
$$

The Lorentz invariance of $\sigma$ implies the invariance of $\mu_{r}$.

Now we comment on some specific representations of this measure, first for $r \geq 0$. In this case the hyperboloid has two sheets which are

$$
V_{r}^{ \pm}=\left\{p \in V_{r}: \pm p^{0}>0\right\}
$$

Lemma 1 For $r \geq 0$, let $f$ have compact support on $V_{r}^{ \pm}$and let $\omega_{r}(p)=\sqrt{|\vec{p}|^{2}+r}$. Then

$$
\int_{V_{r}^{ \pm}} f d \mu_{r}=\int f\left( \pm \omega_{r}(\vec{p}), \vec{p}\right) \frac{d \vec{p}}{2 \omega_{r}(\vec{p})}
$$

Proof. For $V_{r}^{ \pm}$we can take global coordinates $\vec{p}=\left(p^{1}, \ldots, p^{d-1}\right)$. In these coordinates we have

$$
\sigma=\frac{1}{\partial \alpha / \partial p^{0}} d p^{1} \wedge \ldots d p^{d-1}=\frac{1}{2 p^{0}} d p^{1} \wedge \ldots d p^{d-1}
$$

This is the form on $V_{r}^{ \pm}$. Pulling it back to $\mathbb{R}^{d-1}$ with the inverse coordinate function $\phi_{ \pm}(\vec{p})=\left( \pm \omega_{r}(\vec{p}), \vec{p}\right)$ we have

$$
\phi_{ \pm}^{*}(\sigma)=\frac{ \pm 1}{2 \omega(\vec{p})} d p^{1} \wedge \ldots \wedge d p^{d-1}
$$

Now $\phi_{+}$is orientation preserving so $\int_{V_{r}^{+}} f \sigma$ is evaluated as $\int\left(f \circ \phi_{+}\right) \phi_{+}^{*}(\sigma)$. On the other hand $\phi_{-}$is orientation reversing so $\int_{V_{r}^{-}} f \sigma$ is evaluated as $-\int\left(f \circ \phi_{-}\right) \phi_{-}^{*}(\sigma)$ In either case we get the stated result.

Another representation uses light-cone coordinates and now we allow all $r \in \mathbb{R}$. Light-cone coordinates $\hat{p}=\ell(p)$ are defined on $\mathbb{R}^{d}$ by $\hat{p}=\left(p^{-}, \tilde{p}, p^{+}\right)$where $p^{ \pm}=$ $(2)^{-1 / 2}\left(p^{0} \pm p^{d-1}\right)$ and $\tilde{p}=\left(p^{1}, \ldots, p^{d-2}\right)$. For any function $f$ let $\hat{f}=f \circ \ell^{-1}$ be the expression in light-cone coordinates. Also define

$$
V_{r}^{ \pm}=\left\{p \in V_{r}: \pm p^{+}>0\right\}
$$

either in the original coordinates or in light-cone coordinates depending on the context. For $r \geq 0$ these are again the two components of $V_{r}$, but for $r<0$ they are just open sets in $V_{r}$. 
Lemma 2 For any $r$, let $f$ have compact support on $V_{r}^{ \pm}$. Then

$$
\int_{V_{r}^{ \pm}} f d \mu_{r}=\int \hat{f}\left(\frac{|\tilde{p}|^{2}+r}{2 p^{+}}, \tilde{p}, p^{+}\right) \frac{d \tilde{p} d p^{+}}{2\left|p^{+}\right|}
$$

Proof. The integral $\int f \sigma$ can be evaluated as $\int \hat{f} \hat{\sigma}$ where $\hat{\sigma}=\left(\ell^{-1}\right)^{*} \sigma$. Since $\left(\ell^{-1}\right)^{*} \tau=\tau$ we have $d \hat{\alpha} \wedge \hat{\sigma}=\tau$. On $V_{r}^{ \pm}$we can take coordinates $\left(p^{+}, \tilde{p}\right)$. Since $\tau=d p^{-} \wedge d p^{1} \ldots \wedge d p^{d-2} \wedge d p^{+}$and since $\hat{\alpha}(p)=2 p^{+} p^{-}-|\tilde{p}|^{2}$. we find that expressed in these coordinates

$$
\hat{\sigma}=\frac{1}{\partial \hat{\alpha} / \partial p^{-}} d p^{1} \wedge \ldots \wedge d p^{d-2} \wedge d p^{+}=\frac{1}{2 p^{+}} d p^{1} \wedge \ldots \wedge d p^{d-2} \wedge d p^{+}
$$

The inverse coordinate function is $\phi\left(p^{+}, \tilde{p}\right)=\left(\left(|\tilde{p}|^{2}+r\right) / 2 p^{+}, \tilde{p}, p^{+}\right)$defined on the half spaces $\pm p^{+}>0$ in $\mathbb{R}^{d-1}$. The pull back $\phi^{*}(\hat{\sigma})$ to these half spaces has the same form. The function $\phi$ is orientation preserving for $V_{r}^{+}$and orientation reversing for $V_{r}^{-}$. Thus the integral is evaluated as $\pm \int(\hat{f} \circ \phi) \phi^{*}(\hat{\sigma})$ respectively, and in either case we get the stated result.

For $r \leq 0$ the sets $V_{r}^{ \pm}$do not cover all of $V_{r}$. However suppose we define $p_{j}^{ \pm}=$ $(2)^{-1 / 2}\left(p^{0} \pm p^{j}\right)$ with $j=1, \ldots, d-1$ and define more sets $V_{r, j}^{ \pm}=\left\{p \in V_{r}: \pm p_{j}^{+}>0\right\}$ and $\tilde{V}_{r, j}^{ \pm}=\left\{p \in V_{r}: \pm p_{j}^{-}>0\right\}$. On each of these sets we can prove a result similar to (9). These sets do cover $V_{r}$ and by taking a partition of unity subordinate to this covering we can express any integral as a sum of integrals of the type (9).

Lemma 3 Let $f$ be a function on on $\mathbb{R}^{d}-\{0\}$ which is continuous and has compact support. Let $f_{r}$ be the restriction to $V_{r}$. Then

$$
\int f=\int\left(\int_{V_{r}} f_{r} d \mu_{r}\right) d r
$$

Proof. Let $U_{r, j}^{ \pm}=\left\{p \in \mathbb{R}^{d}: \pm p_{j}^{+}>0\right\}$ and $\tilde{U}_{r, j}^{ \pm}=\left\{p \in \mathbb{R}^{d}: \pm p_{j}^{-}>0\right\}$. These cover $\mathbb{R}-\{0\}$ and by introducing a subordinate partition of unity it suffices to prove the theorem assuming that $f$ has compact support in one of these sets, for example $U_{r}^{+}$ defined by $p^{+}=p_{d-1}^{+}>0$. Then $f_{r}$ has compact support in $V_{r}^{+}$for all $r$. We make the change of variables $p^{-} \leftrightarrow r$ with $r=2 p^{+} p^{-}-|\tilde{p}|^{2}$ and then we have

$$
\int \hat{f}\left(p^{-}, \tilde{p}, p^{+}\right) d p^{-} d \tilde{p} d p^{+}=\int \hat{f}\left(\frac{|\tilde{p}|^{2}+r}{2 p^{+}}, \tilde{p}, p^{+}\right) \frac{d \tilde{p} d p^{+} d r}{2\left|p^{+}\right|}=\int\left(\int_{V_{r}} f_{r} d \mu_{r}\right) d r
$$




\section{The single string}

\section{$2.1 \quad$ Pre-constrained}

We now undertake the covariant quantization of the single string. The construction is mostly standard [9]. However, one novelty is that the center of mass momentum is treated as a genuine quantum observable with a distribution of values. Most treatments take a fixed center of mass momentum.

For the open string in $\mathbb{R}^{d}$ the coordinates of the quantum string should be operators $X^{\mu}(\tau, \sigma)$ define for $(\tau, \sigma) \in \mathbb{R} \times[0, \pi]$ and satisfying the wave equation

$$
\left(\frac{\partial^{2} X^{\mu}}{\partial \tau^{2}}-\frac{\partial^{2} X^{\mu}}{\partial \sigma^{2}}\right)=0
$$

with Neumann boundary conditions on $[0, \pi]$. The operators $X^{\mu}$ and the string momentum $P^{\mu}=\partial X^{\mu} / \partial \tau$ are supposed to satisfy the equal $\tau$ commutation relations

$$
\left[X^{\mu}(\sigma, \tau), P^{\nu}\left(\sigma^{\prime}, \tau\right)\right]=i \pi \delta\left(\sigma-\sigma^{\prime}\right) \eta^{\mu \nu}
$$

Corresponding to reparametrization invariance we impose the constraints

$$
\left(\frac{\partial X}{\partial \sigma} \pm \frac{\partial X}{\partial \tau}\right)^{2}=0
$$

That is we ask for states which are annihilated by these operators. Finally we ask that the center of mass $x^{\mu}=\pi^{-1} \int_{0}^{\pi} X^{\mu}(\tau, \sigma) d \sigma$ be parametrized in a forward moving direction. This means we require that the constant center of mass momentum $p^{\mu}=$ $d x^{\mu} / d \tau=\pi^{-1} \int_{0}^{\pi} P^{\mu}(\tau, \sigma) d \sigma$ should satisfy

$$
p^{0}=\frac{d x^{0}}{d \tau}>0
$$

Classically one can find solutions of the wave equation by expanding in eigenfunctions of the Laplacian with Neuman boundary conditions, that is in a cosine series. One finds that

$$
X^{\mu}(\sigma, \tau)=x^{\mu}+p^{\mu} \tau+i \sum_{n \neq 0} \alpha_{n}^{\mu} e^{-i n \tau} \frac{\cos n \sigma}{n}
$$

The quantum operator will be given by the same expression. It formally satisfies the commutation relations (14) if we ask that $x^{\mu}, p^{\mu}, \alpha_{n}^{\mu}$ be operators satisfying the commutation relations

$$
\begin{aligned}
{\left[x^{\mu}, p^{\nu}\right] } & =i \eta^{\mu \nu} \\
{\left[\alpha_{m}^{\mu}, \alpha_{n}^{\nu}\right] } & =m \delta_{m+n} \eta^{\mu \nu}
\end{aligned}
$$


Here is a construction of these operators. First consider the Hilbert space $\mathcal{L}^{2}\left(\mathbb{R}^{d}\right)$. On this space let $x^{\mu}$ be the multiplication operator, let $p_{\nu}=-i \partial / \partial x^{\nu}$ and let $p^{\nu}=$ $\sum_{\nu} \eta^{\mu \nu} p_{\nu}$ (the spacetime representation), or else let $p^{\nu}$ be the multiplication operators and let $x_{\mu}=i \partial / \partial p^{\mu}$, etc. (the momentum representation). In either case these satisfy (18). A convenient dense domain for these operators is $\mathcal{S}\left(\mathbb{R}^{d}\right)$, the Schwartz space of smooth rapidly decreasing functions. This is invariant under the Fourier transform and connects the two representations.

Next let $\ell^{2}=\ell^{2}\left(\mathbb{N}, \mathbb{C}^{d}\right)$ be the space of square summable maps $f: \mathbb{N} \rightarrow \mathbb{C}^{d}$. This has the usual inner product $(f, g)$ and also an indefinite inner product $\langle f, g\rangle$. They are

$$
\begin{gathered}
(f, g)=\sum_{n=1}^{\infty} \sum_{\mu} \overline{f_{\mu n}} g_{\mu n} \\
<f, g>=\sum_{n=1}^{\infty} \sum_{\mu \nu} \eta_{\mu \nu} \overline{f_{\mu n}} g_{\nu n}
\end{gathered}
$$

They are related by $\langle f, g\rangle=(f, J g)$ where $(J g)_{\mu}=\eta_{\mu \mu} g_{\mu}$. (Thus $J=\eta$, but without the geometric interpretation). Let $\mathcal{F}_{j}\left(\ell^{2}\right)$ be the $j$-fold symmetric tensor product of $\ell^{2}$ and let $\mathcal{F}\left(\ell^{2}\right)=\bigoplus_{j=0}^{\infty} \mathcal{F}_{j}\left(\ell^{2}\right)$ be the bosonic Fock space over $\ell^{2}$. Any unitary operator $U$ on $\ell^{2}$ induces a unitary $\otimes^{j} U$ on $\mathcal{F}_{j}\left(\ell^{2}\right)$ and hence an operator $\Gamma(U)$ on $\mathcal{F}\left(\ell^{2}\right)$. We define an indefinite inner product on $\mathcal{F}\left(\ell^{2}\right)$ by $\langle f, g\rangle=(f, \mathcal{J} g)$ where $\mathcal{J}=\Gamma(J)$.

For any operator $\mathcal{O}$ on $\mathcal{F}\left(\ell^{2}\right)$, let $\mathcal{O}^{*}$ be the adjoint with the definite inner product, and let $\mathcal{O}^{\dagger}$ be the adjoint with the indefinite inner product. Hence $(f, \mathcal{O} g)=\left(\mathcal{O}^{*} f, g\right)$ and $\left.\langle f, \mathcal{O} g\rangle=<\mathcal{O}^{\dagger} f, g\right\rangle$. They are related by $\mathcal{O}^{*}=\mathcal{J O}^{\dagger} \mathcal{J}$.

Next define annihilation operators $a(f), b(f)$ on the n-fold symmetric tensor product by

$$
\begin{aligned}
& a(f)\left(f_{1} \otimes \ldots \otimes f_{j}\right)=\sqrt{j}<f, f_{1}>f_{2} \otimes \ldots \otimes f_{j} \\
& b(f)\left(f_{1} \otimes \ldots \otimes f_{j}\right)=\sqrt{j}\left(f, f_{1}\right) f_{2} \otimes \ldots \otimes f_{j}
\end{aligned}
$$

By restriction these define operators on the symmetric subspace and hence on $\mathcal{F}\left(\ell^{2}\right)$. We have $a(f)=b(J f)$. The adjoints satisfy $a^{\dagger}(f)=b^{*}(f)$. We have $\left[b(f), b^{*}(g)\right]=(f, g)$ and $\left[a(f), a^{\dagger}(g)\right]=<f, g>$.

In $\ell^{2}$ there is a standard basis $e_{m}^{\mu}$ defined by $\left(e_{n}^{\mu}\right)_{\nu m}=\delta_{\nu}^{\mu} \delta_{m, n}$. We define for $n>0$

$$
\begin{aligned}
\alpha_{n}^{\mu} & =\sqrt{n} a\left(e_{n}^{\mu}\right) \\
\alpha_{-n}^{\mu} & =\sqrt{n} a^{\dagger}\left(e_{n}^{\mu}\right)
\end{aligned}
$$

These satisfy (18) since $<e_{n}^{\mu}, e_{m}^{\nu}>=\eta^{\mu \nu} \delta_{n, m}$. Let $\mathcal{D}_{0}$ be the dense subspace of $\mathcal{F}\left(\ell^{2}\right)$ generated by applying a finite number of operators $\alpha_{-n}^{\mu}$ to the no excitation state $\Omega_{0}=(1,0,0 \ldots)$. 
Now consider the Hilbert space

$$
\left.\mathcal{L}^{2}\left(\mathbb{R}^{d}\right) \otimes \mathcal{F}\left(\ell^{2}\right)\right) \approx \mathcal{L}^{2}\left(\mathbb{R}^{d}, \mathcal{F}\left(\ell^{2}\right)\right)
$$

Besides the usual inner product this space has an indefinite inner product inherited from $\mathcal{F}\left(\ell^{2}\right)$ and defined by

$$
<\psi, \chi>=\int<\psi(p), \chi(p)>d p
$$

The operators $x^{\mu}, p^{\mu}$ in the momentum representation, and $\alpha_{n}^{\mu}$ all act on this space. Now we can define the coordinate operator $X^{\mu}(\sigma, \tau)$ by (17). It is well defined provided we interpret it as a distribution in $\sigma$ and to restrict a nice domain like $\mathcal{S}\left(\mathbb{R}^{d}\right) \otimes \mathcal{D}_{0}$. It does satisfy (13), (14). On the same domain $\left(x^{\mu}\right)^{\dagger}=x^{\mu},\left(p^{\mu}\right)^{\dagger}=p^{\mu},\left(\alpha_{n}^{\mu}\right)^{\dagger}=\alpha_{-n}^{\mu}$. and hence $\left(X^{\mu}(\sigma, \tau)\right)^{\dagger}=X^{\mu}(\sigma, \tau)$.

We digress to discuss representations of the Lorentz group. First on $\ell^{2}$ there is a representation defined by $(\Lambda f)_{\mu n}=\sum_{\nu} \Lambda_{\mu}{ }^{\nu} f_{\nu n}$ which preserves the indefinite inner product. This induces an operator $\Gamma(\Lambda)$ on $\mathcal{F}\left(\ell^{2}\right)$ which also preserves the indefinite inner product. It is not bounded but is at least defined on vectors with a finite number of entries. Finally for $a \in \mathbb{R}^{d}$ and a proper Lorentz transformation $\Lambda$ we define $U(a, \Lambda)$ on $\mathcal{L}^{2}\left(\mathbb{R}^{d}, \mathcal{F}\left(\ell^{2}\right)\right)$ by

$$
(U(a, \Lambda) \psi)(p)=e^{-i p \cdot a} \Gamma(\Lambda) \psi\left(\Lambda^{-1} p\right)
$$

This is well-defined if $\psi$ takes values in the domain of $\Gamma(\Lambda)$. The operators $U(a, \Lambda)$ give a representation of the inhomogeneous Lorentz group which preserves the indefinite inner product since Lebesgue measure is Lorentz invariant. We note that $U(a, \Lambda)^{-1} a(f) U(a, \Lambda)=a\left(\Lambda^{-1} f\right)$. Since $\Lambda^{-1} e_{n}^{\mu}=\sum_{\nu}\left(\Lambda^{-1}\right)_{\nu}^{\mu} e_{n}^{\nu}=\sum_{\nu} \Lambda_{\nu}^{\mu} e_{n}^{\nu}$ this implies

$$
U(a, \Lambda)^{-1} \alpha_{n}^{\mu} U(a, \Lambda)=\sum_{\nu} \Lambda_{\nu}^{\mu} \alpha_{n}^{\nu}
$$

We also have $x^{\mu} \rightarrow \sum_{\nu} \Lambda_{\nu}^{\mu} x^{\nu}+a^{\mu}$, and $p^{\mu} \rightarrow \sum_{\nu} \Lambda_{\nu}^{\mu} p^{\nu}$ and thus

$$
U(a, \Lambda)^{-1} X^{\mu}(\sigma, \tau) U(a, \Lambda)=\sum_{\nu} \Lambda_{\nu}^{\mu} X^{\nu}(\sigma, \tau)+a^{\mu}
$$

Now we turn to the constraint operators (15). Passing to the Fourier components one finds the operators

$$
\begin{aligned}
L_{0} & =\frac{1}{2} p^{2}+\sum_{n=1}^{\infty} \alpha_{-n} \cdot \alpha_{n} \\
L_{m} & =\alpha_{m} \cdot p+\frac{1}{2} \sum_{n \neq m, 0} \alpha_{m-n} \cdot \alpha_{n} \quad m \neq 0
\end{aligned}
$$


These are well defined on $\mathcal{S}\left(\mathbb{R}^{d}\right) \otimes \mathcal{D}_{0}$ and satisfy $L_{m}^{\dagger}=L_{-m}$.

Instead of asking for states $\psi$ satisfying $L_{n} \psi=0$ for all $n$ we make the standard modification and ask for states satisfying

$$
\begin{aligned}
\left(L_{0}-1\right) \psi & =0 \\
L_{m} \psi & =0 \quad m>0
\end{aligned}
$$

As usual when quantizing a parametrized theory, the dynamics are contained in the constraints. Suppose we define the operator $M^{2}$ (not really a square) on a dense domain in $\mathcal{F}\left(\ell^{2}\right)$ by

$$
M^{2}=2(N-1)
$$

where $N$ is the excitation operator which can be written in any of the following forms

$$
\begin{aligned}
N & =\sum_{n=1}^{\infty} \alpha_{-n} \cdot \alpha_{n} \\
& =\sum_{n, \mu \nu} n \eta_{\mu \nu} a^{\dagger}\left(e_{n}^{\mu}\right) a\left(e_{n}^{\nu}\right) \\
& =\sum_{n, \mu} n b^{*}\left(e_{n}^{\mu}\right) b\left(e_{n}^{\mu}\right)
\end{aligned}
$$

The constraint $\left(L_{0}-1\right) \psi=0$ can be written $\left(p^{2}+M^{2}\right) \psi=0$. In the spacetime representation we have

$$
\left(-\square+M^{2}\right) \psi=0
$$

It the Klein - Gordon equation for an $\mathcal{F}\left(\ell^{2}\right)$ valued function, and gives the evolution in time. The operator $M^{2}$ is identified as a mass operator. The next result shows that $M^{2}$ is self-adjoint and has spectrum $-2,0,2,4,6 \ldots$ with finite multiplicity.

Lemma $4 N$ is self-adjoint and has spectrum $0,1,2, \ldots$ with finite multiplicity.

Proof. Let $\left\{N_{\mu, n}\right\}$ be a finite sequence of positive integers indexed by $\mu=0,1, \ldots, d-1$ and $n=1,2, \ldots$, with at most finitely many $N_{\mu, n} \neq 0$. For each such sequence we define a vector

$$
\psi\left(\left\{N_{\mu, n}\right\}\right)=\prod_{\mu, n} \frac{\left(b^{*}\left(e_{n}^{\mu}\right)\right)^{N_{\mu, n}}}{\sqrt{N_{\mu, n} !}} \Omega_{0}
$$

This is a orthonormal basis for $\mathcal{F}\left(\ell^{2}\right)$ and they are eigenfunctions of $N$ since

$$
N \psi\left(\left\{N_{\mu, n}\right\}\right)=\left(\sum_{\mu, n} n N_{\mu, n}\right) \psi\left(\left\{N_{\mu, n}\right\}\right)
$$

This gives the self adjointness and the spectrum. The finite multiplicity follows since for any positive integer $n^{*}$ there are only a finite number of sequences with $\sum_{\mu, n} n N_{\mu, n}=$ $n^{*}$. 


\subsection{Reconfigured}

The constraint (31) cannot be satisfied in $\mathcal{L}^{2}\left(\mathbb{R}^{d}, \mathcal{F}\left(\ell^{2}\right)\right)$. To impose the constraint we will have to modify the Hilbert space. To begin we write this space as a direct integral over the various mass shells. For the moment our purpose is just to motivate Definition

1 below, so we pass over various technicalities such as the exact definition of the direct integral in this case. (See however Nielsen [17]).

Let $\|\cdot\|$ denote the norm in the Fock space $\mathcal{F}\left(\ell^{2}\right)$ defined by the definite inner product. For $\psi \in \mathcal{L}^{2}\left(\mathbb{R}^{d}, \mathcal{F}\left(\ell^{2}\right)\right)$ the norm squared can be written as

$$
\int_{\mathbb{R}^{d}}\|\psi(p)\|^{2} d p=\int_{-\infty}^{\infty}\left(\int_{V_{r}}\|\psi(p)\|^{2} d \mu_{r}(p)\right) d r
$$

This follows from (12) extended to $\mathcal{L}^{2}$ functions. Then we can make the identification

$$
\mathcal{L}^{2}\left(\mathbb{R}^{d}, \mathcal{F}\left(\ell^{2}\right), d p\right)=\int^{\oplus} \mathcal{L}^{2}\left(V_{r}, \mathcal{F}\left(\ell^{2}\right), d \mu_{r}\right) d r
$$

where $\psi \in \mathcal{L}^{2}\left(\mathbb{R}^{d}, \mathcal{F}\left(\ell^{2}\right), d p\right)$ is identified with the map $r \rightarrow \psi_{r}$ ( the restriction of $\psi$ to $\left.V_{r}\right)$. The indefinite inner product on $\mathcal{F}\left(\ell^{2}\right)$ induces the same on $\mathcal{L}^{2}\left(V_{r}, \mathcal{F}\left(\ell^{2}\right), d \mu_{r}\right)$ and we have, again by (12),

$$
<\psi, \chi>=\int_{-\infty}^{\infty}<\psi_{r}, \chi_{r}>d r
$$

Thus the decomposition can be regarded as a decomposition of indefinite inner product spaces. The operators $M^{2}, L_{m}$ act on $\mathcal{L}^{2}\left(V_{r}, \mathcal{F}\left(\ell^{2}\right), d \mu_{r}\right)$ and we have the decompositions

$$
\begin{aligned}
L_{0}-1 & =\int^{\oplus} \frac{1}{2}\left(-r+M^{2}\right) d r \\
L_{m} & =\int^{\oplus} L_{m} d r
\end{aligned}
$$

This means for example that $\left(L_{m} \psi\right)_{r}=L_{m} \psi_{r}$. Since the Lorentz group acts on $V_{r}$ the operators $U(a, \Lambda)$ act on $\mathcal{L}^{2}\left(V_{r}, \mathcal{F}\left(\ell^{2}\right), d \mu_{r}\right)$, and they preserve the indefinite inner product since the measure is Lorentz invariant. Denoting this representation by $U_{r}(a, \Lambda)$ we have

$$
U(a, \Lambda)=\int^{\oplus} U_{r}(a, \Lambda) d r
$$

To impose the constraint we first make a minimal reconfiguration of the Hilbert space so it can accept the constraints. Since $M^{2}$ has spectrum in $-2,0,2,4, \ldots$ the constraint $\left(L_{0}-1\right) \psi=0$ only has a chance for $r$ in this set. Accordingly we pick out these values from the direct integral and form a direct sum. 
At this stage we also impose the forward moving condition $p_{0}>0$ as much as possible. For $r \geq 0$ we pick out the positive energy part $V_{r}^{+}$of the mass shell. This is not possible for $r<0$ without losing the representation of the Lorentz group, and so we leave it alone.

We these ideas in mind we make the following definitions after (35), (36), (37), (38).

\section{Definition 1}

1. The Hilbert space for the single string is

$$
\begin{aligned}
\mathcal{H} & =\bigoplus_{r=-2,0,2, \ldots} \mathcal{H}_{r} \\
\mathcal{H}_{r} & =\mathcal{L}^{2}\left(V_{r}^{(+)}, \mathcal{F}\left(\ell^{2}\right), d \mu_{r}\right)
\end{aligned}
$$

where $V_{r}^{(+)}=V_{r}^{+}$for $r \geq 0$ and $V_{r}^{(+)}=V_{r}$ for $r<0$.

2. For $\psi, \chi$ in $\mathcal{H}$ with components $\psi_{r}, \chi_{r}$ an indefinite inner product is defined by

$$
<\psi, \chi>=\sum_{r}<\psi_{r}, \chi_{r}>
$$

3. A representation of the inhomogeneous Lorentz group is defined by

$$
U(a, \Lambda)=\bigoplus_{r} U_{r}(a, \Lambda)
$$

4. The constraint operators are defined by

$$
\begin{aligned}
L_{0}-1 & =\bigoplus_{r} \frac{1}{2}\left(-r+M^{2}\right) \\
L_{m} & =\bigoplus_{r} L_{m}
\end{aligned}
$$

Let us be more precise about the domains of $N, M^{2}, L_{m}$. We will define them as closed operators on $\mathcal{H}_{r}$ and then the above equations define them as closed operators on $\mathcal{H}$. As we have noted $N$ (or $M^{2}$ ) is self-adjoint on $\mathcal{F}\left(\ell^{2}\right)$ and we define $N$ on $\mathcal{H}_{r}$ by $(N \psi)(p)=N \psi(p)$ with domain

$$
D(N)=\left\{\psi \in \mathcal{H}_{r}: \psi(p) \in D(N) \text { a.e. } p, \int\|N \psi(p)\|^{2} d \mu_{r}(p)<\infty\right\}
$$

Similarly for each $p$ let $L_{m}(p)$ be the closure of the operator (27) defined on $\mathcal{D}_{0} \subset \mathcal{F}\left(\ell^{2}\right)$. (It is closable since the adjoint $L_{m}^{*}(p)=\mathcal{J} L_{-m} \mathcal{J}$ is densely defined). Then define $L_{m}$ on $\mathcal{H}_{r}$ by $\left(L_{m} \psi\right)(p)=L_{m}(p) \psi(p)$ with domain

$$
D\left(L_{m}\right)=\left\{\psi \in \mathcal{H}_{r}: \psi(p) \in D\left(L_{m}(p)\right) \text { a.e. } p, \int\left\|L_{m}(p) \psi(p)\right\|^{2} d \mu_{r}(p)<\infty\right\}
$$

With these domains we have: 
Lemma $5 N$ and $M^{2}$ are self adjoint, and $L_{m}$ is closed.

Proof. Start with the second. Let $\psi_{j} \in D\left(L_{m}\right)$ satisfy $\psi_{j} \rightarrow \psi$ and $L_{m} \psi_{j} \rightarrow \chi$. Then there exist a subsequence such that for almost every $p$ we have $\psi_{j_{k}}(p) \rightarrow \psi(p)$ and $L_{m}(p) \psi_{j_{k}}(p) \rightarrow \chi(p)$. (This is a corollary of the proof that vector-valued $\mathcal{L}^{p}$ spaces are complete [13]). Since $L_{m}(p)$ is closed it follows that $\psi(p) \in D\left(L_{m}(p)\right)$ and $L_{m}(p) \psi(p)=\chi(p)$ for a.e. $p$. Hence $\psi \in D\left(L_{m}\right)$ and $L_{m} \psi=\chi$. Thus $L_{m}$ is closed

The same argument shows that $N$ is closed. It is also symmetric and since it has a dense set of analytic vectors, for example $\mathcal{C}_{0}^{\infty} \otimes \mathcal{D}_{0}$, it is self-adjoint.

\subsection{Constrained}

Now let $\mathcal{H}^{\prime}$ be the subspace of $\mathcal{H}$ satisfying the constraints (28). We have

$$
\begin{aligned}
\mathcal{H}^{\prime} & =\bigoplus_{r=-2,0,2, \ldots} \mathcal{H}_{r}^{\prime} \\
\mathcal{H}_{r}^{\prime} & =\left\{\psi \in \mathcal{H}_{r}:\left(-r+M^{2}\right) \psi=0, L_{m} \psi=0 \text { for } \mathrm{m}>0\right\}
\end{aligned}
$$

Note that a function $\psi \in \mathcal{H}_{-2}$ is in $\mathcal{H}_{-2}^{\prime}$ iff both $N \psi=0$ and $L_{m} \psi=0$ which is true iff $\psi$ takes values in $\mathcal{F}_{0}\left(\ell^{2}\right) \approx \mathbb{C}$. Thus $\mathcal{H}_{-2}^{\prime}=L^{2}\left(V_{-2}, \mathcal{F}_{0}\left(\ell^{2}\right), d \mu_{-2}\right)$. These are the tachyons.

Next we consider the isotropic or spurious elements in $\mathcal{H}^{\prime}$ which are defined by $\mathcal{H}^{\prime \prime}=\mathcal{H}^{\prime} \cap\left(\mathcal{H}^{\prime}\right)^{\perp}$. Here the orthogonal subspace is defined by the indefinite inner product. Vectors in $\mathcal{H}^{\prime \prime}$ satisfy $\langle\psi, \psi\rangle=0$. The subspace has the form

$$
\begin{aligned}
& \mathcal{H}^{\prime \prime}=\bigoplus_{r} \mathcal{H}_{r}^{\prime \prime} \\
& \mathcal{H}_{r}^{\prime \prime}=\mathcal{H}_{r}^{\prime} \cap\left(\mathcal{H}_{r}^{\prime}\right)^{\perp}
\end{aligned}
$$

Now let $\mathcal{H}^{\text {phys }}=\mathcal{H}^{\prime} / \mathcal{H}^{\prime \prime}$. We identify

$$
\begin{aligned}
& \mathcal{H}^{\text {phys }}=\bigoplus_{r} \mathcal{H}_{r}^{\text {phys }} \\
& \mathcal{H}_{r}^{\text {phys }}=\mathcal{H}_{r}^{\prime} / \mathcal{H}_{r}^{\prime \prime}
\end{aligned}
$$

The indefinite inner product on $\mathcal{H}^{\prime}$ lifts to $\mathcal{H}^{\text {phys }}$ and is the direct sum of the the inner products on $\mathcal{H}_{r}^{\text {phys }}$ lifted from $\mathcal{H}_{r}^{\prime}$.

Now we establish the famous no-ghost theorem. Our method is to reduce the result to a statement pointwise in $p$ and them quote the literature.

Lemma 6 For $d=26,\langle.,$.$\rangle is positive definite on \mathcal{H}_{r}^{\text {phys }}$ and $\mathcal{H}^{\text {phys }}$. 
Proof. It suffices to prove the result on $\mathcal{H}_{r}^{\text {phys }}$. The space $\mathcal{H}_{r}=\mathcal{L}^{2}\left(V_{r}^{(+)}, \mathcal{F}\left(\ell^{2}\right), d \mu_{r}\right)$ can be regarded as a constant fiber direct integral

$$
\mathcal{H}_{r}=\int_{V_{r}^{(+)}}^{\oplus} \mathcal{F}\left(\ell^{2}\right) d \mu_{r}(p)
$$

Indeed the former can be taken as the definition of the latter, so this just amounts to a change in notation. We have the decompositions $\left(-r+M^{2}\right)=\int^{\oplus}\left(-r+M^{2}\right) d \mu_{r}(p)$ and $L_{m}=\int^{\oplus} L_{m}(p) d \mu_{r}(p)$. The constrained space can be characterized as

$$
\begin{aligned}
\mathcal{H}_{r}^{\prime} & =\int_{V_{r}^{(+)}}^{\oplus} \mathcal{H}^{\prime}(p) d \mu_{r}(p) \\
\mathcal{H}^{\prime}(p) & =\left\{\psi \in \mathcal{F}\left(\ell^{2}\right):\left(p^{2}+M^{2}\right) \psi=0, L_{m}(p) \psi=0 \text { for } \mathrm{m}>0\right\}
\end{aligned}
$$

This means $\psi \in \mathcal{H}_{r}^{\prime}$ iff $\psi(p) \in \mathcal{H}^{\prime}(p)$ for a.e. $p \in V_{r}^{(+)}$. Since eigenvalues of $M^{2}$ have finite multiplicity, $\mathcal{H}^{\prime}(p)$ is finite dimensional. Note also $\mathcal{H}^{\prime}(p) \subset \mathcal{D}_{0}$.

Next we have $\left(\mathcal{H}_{r}^{\prime}\right)^{\perp}=\int^{\oplus}\left(\mathcal{H}^{\prime}(p)\right)^{\perp} d \mu_{r}(p)$ and it follows that

$$
\begin{aligned}
\mathcal{H}_{r}^{\prime \prime} & =\int_{V_{r}^{(+)}}^{\oplus} \mathcal{H}^{\prime \prime}(p) d \mu_{r}(p) \\
\mathcal{H}^{\prime \prime}(p) & =\mathcal{H}^{\prime}(p) \cap \mathcal{H}^{\prime}(p)^{\perp}
\end{aligned}
$$

Now for $\psi, \chi \in \mathcal{H}_{r}^{\prime}$ we have $\psi-\chi \in \mathcal{H}_{r}^{\prime \prime}$ if and only if $\psi(p), \chi(p) \in \mathcal{H}^{\prime}(p)$ satisfy $\psi(p)-\chi(p) \in \mathcal{H}^{\prime \prime}(p)$ for a.e. $p$. Thus equivalence classes can be defined pointwise which we write as

$$
\begin{aligned}
\mathcal{H}_{r}^{\text {phys }} & =\int_{V_{r}^{(+)}}^{\oplus} \mathcal{H}^{\text {phys }}(p) d \mu_{r}(p) \\
\mathcal{H}^{\text {phys }}(p) & =\mathcal{H}^{\prime}(p) / \mathcal{H}^{\prime \prime}(p)
\end{aligned}
$$

Now it suffices to prove that the inner product is positive definite on $\mathcal{H}_{\text {phys }}(p)$. A proof of this can be found in Frenkel, Garland, and Zuckerman [6]. They also compute the dimension of this space and show it depends only on $p^{2}$. For the original proofs see Brower [2] and Goddard and Thorn [8].

Lemma $7 U(a, \Lambda)$ determines a unitary representation of the inhomogeneous Lorentz group on $\mathcal{H}^{\text {phys }}$.

Proof. $U_{r}(a, \Lambda)$ is defined on all of $\mathcal{H}_{r}^{\prime}$. It preserves $\mathcal{H}_{r}^{\prime}$ since $\left[L_{m}, U_{r}(a, \Lambda)\right]=0$ by(25). If $\chi \in\left(\mathcal{H}_{r}^{\prime}\right)^{\perp}$ then for $\psi \in \mathcal{H}_{r}^{\prime}$ we have $<\psi, U_{r}(a, \Lambda) \chi>=<U_{r}(a, \Lambda)^{-1} \psi, \chi>=0$ and so $U_{r}(a, \Lambda) \chi \in\left(\mathcal{H}_{r}^{\prime}\right)^{\perp}$. Thus $U_{r}(a, \Lambda)$ preserves $\mathcal{H}_{r}^{\prime \prime}$ and so it lifts to $\mathcal{H}_{r}^{\text {phys }}$. Since it is still inner product preserving it is unitary. 


\subsection{Physical States}

We want to exhibit some non-trivial smooth elements of $\mathcal{H}_{r}^{\prime} \subset \mathcal{L}^{2}\left(V_{r}^{(+)}, \mathcal{F}\left(\ell^{2}\right), d \mu_{r}\right)$. These then determine well-behaved vectors in $\mathcal{H}_{r}^{\text {phys }}$ and $\mathcal{H}^{\text {phys }}$. We start with the fact the for each $p$ the spaces $\mathcal{H}^{\prime}(p)$ are non-trivial (finite dimensional ) vector spaces.

Lemma $8 \Gamma(\Lambda)$ is a bijection from $\mathcal{H}^{\prime}(p)$ to $\mathcal{H}^{\prime}(\Lambda p)$.

Proof. First note that $\Gamma(\Lambda)$ is defined on $\mathcal{H}^{\prime}(p)$ since $\mathcal{H}^{\prime}(p) \subset \mathcal{D}_{0}$. Then we use (25) to conclude that $\Gamma(\Lambda)^{-1} M^{2} \Gamma(\Lambda)=M^{2}$ and that $\Gamma(\Lambda)^{-1} L_{m}(p) \Gamma(\Lambda)=L_{m}\left(\Lambda^{-1} p\right)$. This gives the result.

Lemma 9 For any $q \in V_{r}^{(+)}$there is a neighborhood $U$ of $q$ in $V_{r}^{(+)}$and a smooth family $\Lambda(p, q)$ of Lorentz transformations defined for $p \in U$ such that $\Lambda(p, q) q=p$.

Proof. The proof uses the following well-known fact (see for example [21]). Let $G$ be a Lie group with closed subgroup $H$. Let $\pi: G \rightarrow G / H$ be the projection onto the coset space. Then there are local smooth sections. That is for any point in $G / H$ there is a neighborhood $U$ and a smooth map $s: U \rightarrow G$ such that $\pi \circ s=i d$.

In our case let $\mathcal{L}$ be the proper Lorentz transformations $(\mathcal{L}=$ a component of $S O(d-1,1))$. Fix $q$ and let $H$ be the subgroup which leaves $q$ invariant. (e.g. if $r>0$ then $H=S O(d-1))$. Since $\mathcal{L}$ acts transitively on $V_{r}^{(+)}$we have that the map $\Lambda \rightarrow \Lambda q$ from $\mathcal{L}$ to $V_{r}^{(+)}$lifts to a diffeomorphism $\Lambda H \rightarrow \Lambda q$ from the coset space $\mathcal{L} / H$ to $V_{r}^{(+)}$. This identifies the two spaces.

Now from the general result there is a neighborhood $U$ of $H$ in $\mathcal{L} / H$ and a map $s: U \rightarrow \mathcal{L}$ such that $s(\Lambda H) H=\Lambda H$ for all $\Lambda H \subset U$. Equivalently we can regard $U$ as a neighborhood of $q$ in $V_{r}^{(+)}$and have a map $s: U \rightarrow \mathcal{L}$ satisfying $s(p) q=p$ for all $p \in U$. Defining $\Lambda(p, q)=s(p)$ we have the result.

Lemma 10 Let $q, U$, and $\Lambda(p, q)$ be as above.

1. $\Gamma(\Lambda(p, q))$ is a bijection from $\mathcal{H}^{\prime}(q)$ to $\mathcal{H}^{\prime}(p)$.

2. There exist $\psi \in \mathcal{C}^{\infty}\left(U, \mathcal{F}\left(\ell^{2}\right)\right)$ such that $\psi(p) \in \mathcal{H}^{\prime}(p)$ for all $p \in U$.

3. There exist $\psi \in \mathcal{C}_{0}^{\infty}\left(V_{r}^{(+)}, \mathcal{F}\left(\ell^{2}\right)\right)$ such that $\psi(p) \in \mathcal{H}^{\prime}(p)$ for all $p \in V_{r}$, i.e. $\psi \in \mathcal{H}_{r}^{\prime}$.

Proof. The first follows from lemma 8. For the second let $\psi_{0} \in \mathcal{C}_{0}^{\infty}\left(U, \mathcal{H}^{\prime}(q)\right)$ and take $\psi(p)=\Gamma(\Lambda(p, q)) \psi_{0}(p)$. Multiplying by $\chi \in \mathcal{C}_{0}^{\infty}(U)$ gives a functions satisfying the third condition. By adding functions for different neighborhoods $U$ we get a rich class of functions. 


\section{Remarks.}

1. There is another way to look at this result. Let $\xi$ the set of all pairs $\{(p, \psi)\}$ where $p \in V_{r}$ and $\psi \in \mathcal{H}^{\prime}(p)$, and let $\xi_{U}$ be the subset of pairs with $p \in U$. We have smooth mappings $(p, \psi) \rightarrow\left(p, \Gamma\left(\Lambda(p, q)^{-1}\right) \psi\right)$ from $\xi_{U}$ to $U \times \mathcal{H}^{\prime}(q)$ and hence from $\xi_{U}$ to $U \times \mathbb{C}^{s}$ where $s$ is the dimension of $\mathcal{H}^{\prime}(q)$. Covering $V_{r}^{(+)}$by such charts gives $\xi$ the structure of a smooth vector bundle. Such bundles have smooth sections which our result.

2. If $r>0$ then the neighborhood $U$ can be taken to be all of $V_{r}^{+}$. In this case we can take $q=(\sqrt{r}, 0,0, .$.$) and let \Lambda(p, q)$ be the standard boost to $p$. ( See Weinberg [22], equation (2.5.24) for the explicit formula).

\section{$3 \quad$ String field theory}

We develop the string field theory by taking the dynamical equation satisfied by the single string wave equation, specializing to real solutions, treating these solutions as a classical Hamiltonian system, quantizing this system, and then finally imposing the constraints. This is "second quantization", and the exact status of this process is always a little ambiguous ... which quantization was the genuine quantization? Whatever attitude one takes one ends up at the same place. In any case the quantization process is just meant to be suggestive of a true quantum theory.

Our formulation of the problem uses techniques which have been useful in the study of quantum field theory in curved spacetime [14], [3], [4].

\subsection{String field equation}

We start by defining real elements of $\mathcal{F}\left(\ell^{2}\right)$. These are vectors satisfying $C \psi=\psi$ where $C$ is some conjugation on $\mathcal{F}\left(\ell^{2}\right)$. A conjugation is an anti-linear isometry satisfying $C^{2}=1$. We also want our conjugation to satisfy $\left[C, M^{2}\right]=0$ and $[C, \mathcal{J}]=0$. Then also $\overline{\langle\psi, \chi\rangle}=\left\langle C \psi, C \chi>\right.$. For example one could take $C_{0}=\Gamma\left(c_{0}\right)$ where $c_{0}$ is the usual conjugation $c_{0} \psi=\bar{\psi}$ on $\ell^{2}$. In the following we just suppose that some $C$ satisfying the above conditions has been chosen.

Now we study the Klein-Gordon equation:

$$
\left(-\square+M^{2}\right) U=0
$$

for functions $U: \mathbb{R}^{d} \rightarrow \mathcal{F}\left(\ell^{2}\right)$. Given real $F_{0}, G_{0} \in \mathcal{C}_{0}^{\infty}\left(\mathbb{R}^{d-1}, \mathcal{F}\left(\ell^{2}\right)\right)$ there is a unique smooth real solution $U$ such that $U=F_{0}$ and $\partial U / \partial x^{0}=G_{0}$ on some surface $x^{0}=t_{0}$, called a Cauchy surface. The solution has compact support on any other Cauchy surface $x^{0}=t$. Such solutions will be called regular. 
Associated with this equation there is a real bilinear form. For any functions $U(x)=$ $U\left(x^{0}, \vec{x}\right)$ and $V(x)=V\left(x^{0}, \vec{x}\right)$ it is defined by

$$
\sigma_{t}(U, V)=\int_{x_{0}=t}\left(<U(x), \frac{\partial V}{\partial x^{0}}(x)>-<\frac{\partial U}{\partial x^{0}}(x), V(x)>\right) d \vec{x}
$$

Green's identity states that for $t>s$

$$
\begin{aligned}
& \sigma_{t}(U, V)-\sigma_{s}(U, V)= \\
& \quad \int_{s<x^{0}<t}\left(<U(x),\left(\left(-\square+M^{2}\right) V\right)(x)>-<\left(\left(-\square+M^{2}\right) U\right)(x), V(x)>\right) d x
\end{aligned}
$$

Thus if $U, V$ are regular solutions then $\sigma_{t}(U, V)$ is independent of $t$ and is just denoted $\sigma(U, V)$. This form is skew symmetric and non-degenerate on the space of regular solutions, i.e. it is symplectic. This symplectic form will be the basis on quantization.

But first we develop some facts about fundamental solutions for our KG equation. The following results are standard for the scalar KG equation. Advanced and retarded fundamental solutions $E^{ \pm}$are operators on functions $F \in \mathcal{C}_{0}^{\infty}\left(\mathbb{R}^{d}, \mathcal{F}\left(\ell^{2}\right)\right)$ defined by

$$
\left(E^{ \pm} F\right)(x)=\frac{1}{(2 \pi)^{d / 2}} \int_{\Gamma_{ \pm} \times \mathbb{R}^{d-1}} \frac{e^{i p \cdot x}}{p^{2}+M^{2}} \tilde{F}(p) d p
$$

The $p^{0}$ contour $\Gamma_{ \pm}$is the real line shifted slightly above/below the real axis. The exact choice does not matter because $\tilde{F}$ is entire and rapidly decreasing in real directions. Also for $x \in \mathbb{R}^{d}$ let $J^{ \pm}(x)=\left\{y:(x-y)^{2}<0, \pm\left(x^{0}-y^{0}\right)>0\right\}$ be the past or future of $x$. For $A \subset \mathbb{R}^{d}$ define $J^{ \pm}(A)=\cup_{x \in A} J^{ \pm}(x)$.

Lemma 11 For $F \in \mathcal{C}_{0}^{\infty}\left(\mathbb{R}^{d}, \mathcal{F}\left(\ell^{2}\right)\right)$ we have $\left(-\square+M^{2}\right) E^{ \pm} F=F$ and $\operatorname{supp}\left(E^{ \pm} F\right) \subset$ $J^{ \pm}(\operatorname{supp} F)$.

Proof. Let $P_{r}$ be the operator on $\mathcal{F}\left(\ell^{2}\right)$ which is the projection onto the eigenspace $M^{2}=r$. On the range of $P_{r}$ we have $E^{ \pm} F=E_{r}^{ \pm} F$ where

$$
\left(E_{r}^{ \pm} F\right)(x)=\frac{1}{(2 \pi)^{d / 2}} \int_{\Gamma_{ \pm} \times \mathbb{R}^{d-1}} \frac{e^{i p \cdot x}}{p^{2}+r} \tilde{F}(p) d p
$$

These are the advanced/retarded fundamental solutions for the Klein-Gordon equation with mass $r$ and they satisfy $(-\square+r) E_{r}^{ \pm} F=F$ and $\operatorname{supp}\left(E_{r}^{ \pm} F\right) \subset J^{ \pm}(\operatorname{supp} F)$.

Now $E^{ \pm}$are fundamental solutions since $P_{r}\left(-\square+M^{2}\right) E^{ \pm} F=(-\square+r) E_{r}^{ \pm} P_{r} F=$ $P_{r} F$. For the support property we have $\operatorname{supp}\left(E^{ \pm} F\right) \subset \cup_{r} \operatorname{supp}\left(P_{r} E^{ \pm} F\right)$. But $P_{r} E^{ \pm} F=$ $E_{r}^{ \pm} P_{r} F$ and $\operatorname{supp}\left(E_{r}^{ \pm} P_{r} F\right) \subset J^{ \pm}\left(\operatorname{supp}\left(P_{r} F\right)\right) \subset J^{ \pm}(\operatorname{supp} F)$ and hence the result.

The propagator function is defined by $E=E^{+}-E^{-}$. Then $U=E F$ is a regular solution. In fact we have: 


\section{Lemma 12}

1. $U$ is a regular solution of $\left(-\square+M^{2}\right) U=0$ iff it can be written $U=E F$ with $F \in \mathcal{C}_{0}^{\infty}\left(\mathbb{R}^{d}, \mathcal{F}\left(\ell^{2}\right)\right)$

2. $F \in \mathcal{C}_{0}^{\infty}\left(\mathbb{R}^{d}, \mathcal{F}\left(\ell^{2}\right)\right)$ satisfies $E F=0$ iff $F=\left(-\square+M^{2}\right) H$ for some $H \in$ $\mathcal{C}_{0}^{\infty}\left(\mathbb{R}^{d}, \mathcal{F}\left(\ell^{2}\right)\right)$

Proof. Let $U$ be a regular solution and let $\theta=\theta\left(x^{0}\right)$ be smooth and satisfy $\theta=1$ for $x^{0}$ sufficiently positive and $\theta=0$ for $x^{0}$ sufficiently negative. Define $F=\left(-\square+M^{2}\right)(\theta U)=$ $-\left(-\square+M^{2}\right)((1-\theta) U)$. Then $F$ has compact support. Next note that $\theta U=E^{+} F$ since the difference satisfies the $K G$ equation and vanishes in the distant past. Similarly $-((1-\theta) U)=E^{-} F$. Taking the difference of the last two equations gives $U=E F$. This proves the first claim.

For the second suppose $F=\left(-\square+M^{2}\right) H$. Then $E F=\left(-\square+M^{2}\right) E H=0$. On the other hand if $E F=0$, then $H=E^{ \pm} F$ has compact support and $\left(-\square+M^{2}\right) H=F$.

The next identity establishes a connection between any solution and its values on any Cauchy surface (all in the sense of distributions). We define

$$
<U, F>=\int<U(x), F(x)>d x
$$

Lemma 13 For $F \in \mathcal{C}_{0}^{\infty}\left(\mathbb{R}^{d}, \mathcal{F}\left(\ell^{2}\right)\right)$ and any regular solution $U$ :

$$
\sigma(U, E F)=<U, F>
$$

or equivalently For $F, G \in \mathcal{C}_{0}^{\infty}\left(\mathbb{R}^{d}, \mathcal{F}\left(\ell^{2}\right)\right)$

$$
\sigma(E F, E G)=<E F, G>=-<F, E G>
$$

Proof. By Green's identity we have for $t<0$

$$
\sigma_{0}\left(U, E^{+} F\right)-\sigma_{t}\left(U, E^{+} F\right)=\int_{t<x^{0}<0}<U(x), F(x)>d x
$$

Letting $t \rightarrow-\infty$ we get an expression for $\sigma_{0}\left(U, E^{+} F\right)$. Similarly we get an expression for $\sigma_{0}\left(U, E^{-} F\right)$. They are

$$
\sigma_{0}\left(U, E^{ \pm} f\right)= \pm \int_{\mp x^{0}>0}<U(x), F(x)>d x
$$

Take the difference to obtain the result. 
We next want to make a connection with the single string Hilbert space $\mathcal{H}=\oplus_{r} \mathcal{H}_{r}$. Given $F \in \mathcal{C}_{0}^{\infty}\left(\mathbb{R}^{d}, \mathcal{F}\left(\ell^{2}\right)\right)$ we define $\Pi F \in \mathcal{H}$ by specifying that $(\Pi F)_{r} \in \mathcal{H}_{r}$ is obtained by taking the Fourier transform, projecting onto the subspace $M^{2}=r$ with $P_{r}$, and then restricting to $V_{r}^{(+)}$. More precisely for $p \in V_{r}^{(+)}$we define

$$
(\Pi F)_{r}(p)=\sqrt{2 \pi} P_{r} \tilde{F}(p)
$$

We will need to exclude tachyons, so we restrict to functions $F$ which take values in

$$
\mathcal{F}_{+}\left(\ell^{2}\right) \equiv\left(\mathcal{F}_{0}\left(\ell^{2}\right)\right)^{\perp} \equiv \bigoplus_{j \geq 1} \mathcal{F}_{j}\left(\ell^{2}\right)
$$

We have $M^{2} \geq 0$ on this subspace. Hence for such $F,(\Pi F)_{-2}=0$ and hence $\Pi F \in \mathcal{H}_{+}$ the no-tachyon subspace of $\mathcal{H}$ :

$$
\mathcal{H}_{+}=\bigoplus_{r \geq 0} \mathcal{H}_{r}
$$

Lemma 14 For (real) $F, G \in \mathcal{C}_{0}^{\infty}\left(\mathbb{R}^{d}, \mathcal{F}_{+}\left(\ell^{2}\right)\right)$

$$
\sigma(E F, E G)=<E F, G>=2 \operatorname{Im}<\Pi F, \Pi G>
$$

Proof. Only the second identity needs proof. We compute with $E_{r}=E_{r}^{+}-E_{r}^{-}$

$$
\begin{aligned}
<E F, G> & =\sum_{r \geq 0}<E_{r} P_{r} F, P_{r} G> \\
& =\sum_{r \geq 0} 2 \operatorname{Im}<(\Pi F)_{r},(\Pi G)_{r}>=2 \operatorname{Im}<\Pi F, \Pi G>
\end{aligned}
$$

The second step follows since for $r \geq 0$ and any $F, G$

$$
\begin{aligned}
<E_{r} F, G> & =-\int_{\Gamma_{+}-\Gamma_{-}}<\tilde{F}(\bar{p}), \tilde{G}(p)>\frac{1}{p^{2}+r} d p \\
& =-2 \pi i\left\{\int<\tilde{F}\left(\omega_{r}(\vec{p}), \vec{p}\right), \tilde{G}\left(\omega_{r}(\vec{p}), \vec{p}\right)>\frac{d \vec{p}}{2 \omega_{r}(\vec{p})}-c . c .\right\} \\
& =2 \operatorname{Im}<\sqrt{2 \pi} \tilde{F}\left|V_{r}^{+}, \sqrt{2 \pi} \tilde{G}\right| V_{r}^{+}>
\end{aligned}
$$

In the second step we have evaluated the contour integral by taking residues at $p^{0}=$ $\pm \omega(\vec{p})$. We have also used $\overline{<\tilde{F}(p), \tilde{G}(p)>}=<\mathcal{C} \tilde{F}(p), \mathcal{C} \tilde{G}(p)>=<\tilde{F}(-p), \tilde{G}(-p)>$ for $p$ real, a consequence of the reality of $F, G$. 


\subsection{String field operator}

Now we quantize solutions of the string field equation. We take as our phase space the space of all regular solutions $\Phi$ of $\left(-\square+M^{2}\right) \Phi=0$ with symplectic form $\sigma\left(\Phi, \Phi^{\prime}\right)$ defined previously. For each solution $U$ there is a function $\Phi \rightarrow \sigma(\Phi, U)$ on the phase space. We quantize these functions by replacing them by operators on a complex Hilbert space, also denoted $\sigma(\Phi, U)$, which are required to satisfy

$$
[\sigma(\Phi, U), \sigma(\Phi, V)]=i \sigma(U, V)
$$

This looks more familiar if we identify solutions with their data on some Cauchy surface. Then the operators are $\sigma\left(\Phi_{0}, \Pi_{0} ; F_{0}, G_{0}\right)=\Phi_{0}\left(G_{0}\right)-\Pi_{0}\left(F_{0}\right)$ and the commutator is written $\left[\sigma\left(\Phi_{0}, \Pi_{0} ; F_{0}, G_{0}\right), \sigma\left(\Phi_{0}, \Pi_{0} ; F_{0}^{\prime}, G_{0}^{\prime}\right)\right]=i \sigma\left(F_{0}, G_{0} ; F_{0}^{\prime}, G_{0}^{\prime}\right)$. As a special case we have the standard $\left[\Phi_{0}\left(G_{0}\right), \Pi_{0}\left(F_{0}\right)\right]=i<G_{0}, F_{0}>$.

The full spacetime field operator is obtained from the operators $\sigma(\Phi, U)$ just as in the classical case. Following (58) we define the field operator as a distribution by

$$
\Phi(F)=\sigma(\Phi, E F)
$$

Then $\Phi(F)$ satisfies the field equation and has a local commutator as the next result shows.

Lemma 15 let $\sigma(\Phi, U)$ be a family of operators indexed by regular solutions $U$ of the $K G$ equation and satisfying (68). Then the operators $\Phi(F)=\sigma(\Phi, E F)$ defined for $F \in \mathcal{C}_{0}^{\infty}\left(\mathbb{R}^{d}, \mathcal{F}\left(\ell^{2}\right)\right)$ satisfies

$$
\begin{aligned}
\Phi\left(\left(-\square+M^{2}\right) F\right) & =0 \\
{[\Phi(F), \Phi(G)] } & =-i<F, E G>
\end{aligned}
$$

Furthermore every operator valued distribution $\Phi(F)$ satisfying (70) arises in this way.

Proof. The field equation follows from $E\left(-\square+M^{2}\right) F=0$ and the commutator follows from the identity (59). For the converse given $\Phi(F)$ we define $\sigma(\Phi, U)=\Phi(F)$ for any $F$ such that $U=E F$. To see that this is well defined we have to show that if $E F_{1}=E F_{2}$ then $\Phi\left(F_{1}\right)=\Phi\left(F_{2}\right)$, or if $E F=0$ then $\Phi(F)=0$. But we have seen that $E F=0$ implies $F=\left(-\square+M^{2}\right) H$ and hence the result follows. The operators $\sigma(\Phi, U)$ have the commutator (68) again by the identity (59).

\section{Remarks.}

1. Since $\sigma(U, V)$ is a symplectic form, representations of (68) do exist on general principles. Thus string field theories exist. Furthermore the spacetime field $\Phi(F)$ defined by (69) is strictly local because if $\operatorname{supp}(F)$ and $\operatorname{supp}(G)$ are spacelike separated, then $\operatorname{supp}(E F)$ and $\operatorname{supp}(G)$ do not overlap and hence $[\Phi(F), \Phi(G)]=$ $-i<F, E G>=0$. All this holds without suppressing the negative mass part of the equation! 
2. However this is not the end of the story. We actually want the particular representation in which time translation is unitarily implemented with positive energy. (One can think of this as the forward moving condition again). Choosing a particular representation requires a complex structure or a "one-particle structure" on phase space. These are equivalent to expressing the symplectic form $\sigma(E F, E G)$ as the imaginary part of an inner product on some complex Hilbert space. But if we suppress the tachyon then this has already been accomplished in (65) where it is written as $2 \operatorname{Im}<\Pi F, \Pi G\rangle$. Furthermore it is this choice which is associated with positive energy as we shall see.

These considerations lead to the following definition. Tachyons are completely suppressed. We bypass $\sigma(\Phi, U)$ and go directly to operators $\Phi(F)$ satisfying (70). Also we enlarge the class of test functions from compact support to the Schwartz space of smooth rapidly decreasing functions. The Hilbert space is the Fock space over the no-tachyon single string Hilbert space $\mathcal{H}_{+}$:

$$
\mathcal{K}=\mathcal{F}\left(\mathcal{H}_{+}\right)
$$

This has the indefinite inner product $\langle\Psi, \Xi\rangle=(\Psi, \Gamma(\mathcal{J}) \Xi)$. States in $\mathcal{K}$ with finitely many entries are denoted $\mathcal{K}_{f}$.

Definition 2 (The String Field). For $F \in \mathcal{S}\left(\mathbb{R}^{d}, \mathcal{F}_{+}\left(\ell^{2}\right)\right)$ we have $\Pi F \in \mathcal{H}_{+}$and we define on $\mathcal{K}_{f}$

$$
\Phi(F)=a^{\dagger}(\Pi F)+a(\Pi F)
$$

\section{Theorem 1}

1. The string field satisfies $\Phi(F)^{\dagger}=\Phi(F)$, the field equation $\Phi\left(\left(-\square+M^{2}\right) F\right)=0$, and has the commutator $[\Phi(F), \Phi(G)]=-i<F, E G\rangle$.

2. There is positive energy representation $\mathcal{U}(a, \Lambda)$ of the inhomogeneous Lorentz group on $\mathcal{K}$ such that

$$
\mathcal{U}(a, \Lambda) \Phi(F) \mathcal{U}(a, \Lambda)^{-1}=\Phi\left(F_{a, \Lambda}\right)
$$

where $F_{a, \Lambda}(x)=\Gamma(\Lambda) F\left(\Lambda^{-1}(x-a)\right)$.

Proof. The field equation is satisfied since $\Pi\left(-\square+M^{2}\right) F=0$. The commutator is evaluated as

$$
[\Phi(F), \Phi(G)]=2 i \operatorname{Im}<\Pi F, \Pi G>=-i<F, E G>
$$


since the identity (65) holds for $\mathcal{S}$ as well as $\mathcal{C}_{0}^{\infty}$. The representation is defined by $\mathcal{U}(a, \Lambda)=\Gamma(U(a, \Lambda))$. We compute

$$
\begin{aligned}
\mathcal{U}(a, \Lambda) \Phi(F) \mathcal{U}(a, \Lambda)^{-1} & =a^{\dagger}(U(a, \Lambda) \Pi F)+a(U(a, \Lambda) \Pi F) \\
& =a^{\dagger}\left(\Pi F_{a, \Lambda}\right)+a\left(\Pi F_{a, \Lambda}\right) \\
& =\Phi\left(F_{a, \Lambda}\right)
\end{aligned}
$$

As noted we have the following corollary:

Corollary 1 (Locality). If $F, G$ have spacelike separated supports $[\Phi(F), \Phi(G)]=0$.

Now we impose the constraint, and just as for the single string this will give us a positive definite inner product. Let $\hat{L}_{m}$ be the Fourier transform of $L_{m}$, that is $\hat{L}_{m}$ is given by (27) but with $p_{\mu}=-i \partial / \partial x^{\mu}$. We would like to select states which are annihilated by $L_{m} \Phi$ for $m>0$. However, just as for the Gupta- Beuler quantization of the electromagnetic field [19] we must compromise and only impose the condition on the negative frequency part of the field defined by $\Phi_{-}(F)=a(\Pi F)$. This is defined and anti-linear on complex test functions. We look for states annihilated by $\left(\hat{L}_{m} \Phi_{-}\right)(F) \equiv$ $\Phi_{-}\left(\hat{L}_{-m} F\right)$. This is fulfilled by taking the subspace

$$
\mathcal{K}^{\prime}=\mathcal{F}\left(\mathcal{H}_{+}^{\prime}\right) \quad \mathcal{H}_{+}^{\prime}=\mathcal{H}^{\prime} \cap \mathcal{H}_{+}
$$

All $\Psi \in \mathcal{K}^{\prime}$ satisfy the required $a\left(\Pi \hat{L}_{-m} F\right) \Psi=0$ since if $\psi \in \mathcal{H}_{+}^{\prime}$ then

$$
<\Pi \hat{L}_{-m} F, \psi>=<L_{-m} \Pi F, \psi>=<\Pi F, L_{m} \psi>=0
$$

Thus $\mathcal{K}^{\prime}$ is our constrained space, something we might have guessed directly.

Next let $\mathcal{K}^{\prime \prime}=\mathcal{K}^{\prime} \cap\left(\mathcal{K}^{\prime}\right)^{\perp}$ be the isotropic vectors in $\mathcal{K}^{\prime}$. and define

$$
\mathcal{K}^{\text {phys }}=\mathcal{K}^{\prime} / \mathcal{K}^{\prime \prime}
$$

This space inherits an indefinite inner product from $\mathcal{K}^{\prime}$.

Lemma 16 For $d=26$ the inner product on $\mathcal{K}^{\text {phys }}$ is positive definite and we have the identification of Hilbert spaces:

$$
\mathcal{K}^{\text {phys }}=\mathcal{F}\left(\mathcal{H}_{+}^{\text {phys }}\right) \quad \mathcal{H}_{+}^{\text {phys }}=\mathcal{H}_{+}^{\prime} / \mathcal{H}_{+}^{\prime \prime}
$$

Proof. $\mathcal{H}_{+}^{\prime \prime}=\mathcal{H}^{\prime \prime} \cap \mathcal{H}_{+}$is a closed subspace of $\mathcal{H}_{+}^{\prime}$ and so we can write $\mathcal{H}_{+}^{\prime}=\mathcal{H}_{+}^{\prime \prime} \oplus \mathcal{M}$ where $\mathcal{M}$ is the orthogonal complement with respect to the definite inner product. The projection onto $\mathcal{M}$ has kernel $\mathcal{H}_{+}^{\prime \prime}$ and gives an identification of $\mathcal{M}$ with $\mathcal{H}_{+}^{\text {phys }}$ which preserves the indefinite inner product. Thus the inner product is positive definite on $\mathcal{M}$, and of course zero if either entry is in $\mathcal{H}_{+}^{\prime \prime}$. 
Now we have the identification of Hilbert spaces [7]

$$
\mathcal{K}^{\prime}=\mathcal{F}\left(\mathcal{H}_{+}^{\prime \prime} \oplus \mathcal{M}\right)=\mathcal{F}\left(\mathcal{H}_{+}^{\prime \prime}\right) \otimes \mathcal{F}(\mathcal{M})
$$

Under this identification $\Gamma(\mathcal{J})=\Gamma(\mathcal{J}) \otimes \Gamma(\mathcal{J})$ and so the induced indefinite inner product satisfies $<\Psi_{1} \otimes \Psi_{2}, \Psi_{1}^{\prime} \otimes \Psi_{2}^{\prime}>=<\Psi_{1}, \Psi_{1}^{\prime}><\Psi_{2}, \Psi_{2}^{\prime}>$. Splitting $\mathcal{F}\left(\mathcal{H}_{+}^{\prime \prime}\right)=$ $\mathcal{F}_{0}\left(\mathcal{H}_{+}^{\prime \prime}\right) \oplus \mathcal{F}_{+}\left(\mathcal{H}_{+}^{\prime \prime}\right)$ and using $\mathcal{F}_{0}\left(\mathcal{H}_{+}^{\prime \prime}\right) \approx \mathbb{C}$ we have

$$
\mathcal{K}^{\prime}=\mathcal{F}(\mathcal{M}) \oplus\left(\mathcal{F}_{+}\left(\mathcal{H}_{+}^{\prime \prime}\right) \otimes \mathcal{F}(\mathcal{M})\right)
$$

with the natural indefinite inner product. Every component of $\mathcal{F}_{+}\left(\mathcal{H}_{+}^{\prime \prime}\right) \otimes \mathcal{F}(\mathcal{M})$ has at least one factor in $\mathcal{H}_{+}^{\prime \prime}$ and so we can identify

$$
\mathcal{K}^{\prime \prime}=\mathcal{F}_{+}\left(\mathcal{H}_{+}^{\prime \prime}\right) \otimes \mathcal{F}(\mathcal{M})
$$

Thus

$$
\mathcal{K}^{\text {phys }}=\mathcal{F}(\mathcal{M})=\mathcal{F}\left(\mathcal{H}_{+}^{\text {phys }}\right)
$$

These identifications preserve the indefinite inner product. Since the inner product is positive definite on $\mathcal{H}_{+}^{\text {phys }}$ it is positive definite on $\mathcal{K}^{\text {phys }}$.

For certain test functions the string field operator $\Phi(F)$ on $\mathcal{K}$ determines an operator on $\mathcal{K}^{\text {phys }}$. We define

Definition $3 F \in \mathcal{S}\left(\mathbb{R}^{d}, \mathcal{F}_{+}\left(\ell^{2}\right)\right)$ is a constrained test function if $\Pi F \in \mathcal{H}_{+}^{\prime}$

To get real constrained test functions it is useful to pick a particular conjugation on $\mathcal{F}\left(\ell^{2}\right)$. It is $C_{1}=\Gamma\left(c_{1}\right)$ where $c_{1}$ on $\ell^{2}$ is defined by $\left(c_{1} f\right)_{0 n}=\bar{f}_{0 n}$ and $\left(c_{1} f\right)_{k n}=-\bar{f}_{k n}$ for $k=1, \ldots, d-1$. For the next result real means $C_{1} \psi=\psi$.

Lemma 17 Non-trivial (real) constrained test functions exist

Proof. Take $r \geq 0$ and choose $\psi_{0} \in \mathcal{C}_{0}^{\infty}\left(V_{r}^{+}, \mathcal{F}\left(\ell^{2}\right)\right)$ so that $\psi_{0}(p) \in \mathcal{H}^{\prime}(p)$ for all $p \in V_{r}^{+}$. Then $\psi$ defines an element of $\mathcal{H}_{r}^{\prime}$ and hence an element of $\mathcal{H}_{+}^{\prime}$. We have seen that such functions exist in lemma 10.

Next define

$$
\psi\left(\omega_{r}(\vec{p}), \vec{p}\right)=\psi_{0}\left(\omega_{r}(\vec{p}), \vec{p}\right)+C_{1} \psi_{0}\left(\omega_{r}(\vec{p}),-\vec{p}\right)
$$

This satisfies $C_{1} \psi\left(\omega_{r}(\vec{p}), \vec{p}\right)=\psi\left(\omega_{r}(\vec{p}),-\vec{p}\right)$ and is still an element of $\mathcal{H}_{r}^{\prime}$. This is so since $C_{1} \alpha_{n}^{0} C_{1}=\alpha_{n}^{0}$ and $C_{1} \alpha_{n}^{k} C_{1}=-\alpha_{n}^{k}$ and hence $C_{1} L_{m}\left(\omega_{r}(\vec{p}), \vec{p}\right) C_{1}=L_{m}\left(\omega_{r}(\vec{p}),-\vec{p}\right)$.

We will find $F$ so that $\Pi F=\psi$. First write $\psi\left(\omega_{r}(\vec{p}), \vec{p}\right)=h(\vec{p})$ for a function $h \in \mathcal{C}_{0}^{\infty}\left(\mathbb{R}^{d-1}, \mathcal{F}\left(\ell^{2}\right)\right)$. (Or $\mathbb{R}^{d-1}-\{0\}$ if $r=0$ ). Let $\chi \in \mathcal{C}_{0}^{\infty}(\mathbb{R})$ be real and satisfy $\chi(0)=1$. We define $F$ by specifying that the Fourier transform be

$$
\tilde{F}\left(p^{0}, \vec{p}\right)=(2 \pi)^{-(1 / 2)} \chi\left(-\left(p^{0}\right)^{2}+|\vec{p}|^{2}+r\right) h(\vec{p})
$$


Then $\tilde{F}$ is smooth and has compact support and hence $F \in \mathcal{S}\left(\mathbb{R}^{d}, \mathcal{F}\left(\ell^{2}\right)\right)$. Since $h(\vec{p}) \in \operatorname{Ran} P_{r}$ we have $(\Pi F)_{r}=\psi$ and $(\Pi F)_{r^{\prime}}=0$ for $r^{\prime} \neq r$ as required. Since elements of $\operatorname{Ran} P_{r}$ have no zero component in Fock space, this is true of $h(\vec{p})$ and hence $F(x)$. Thus $F$ takes values in $\mathcal{F}_{+}$. Finally we have $C_{1} \tilde{F}(p)=\tilde{F}(-p)$ and hence $C_{1} F(x)=F(x)$ so $F$ is real.

Recall that $\mathcal{K}_{f}$ is the subspace of $\mathcal{K}$ with a finite number of entries. Similarly define $\mathcal{K}_{f}^{\prime}$ and $\mathcal{K}_{f}^{\prime \prime}$ and $\mathcal{K}_{f}^{\text {phys }}=\mathcal{K}_{f}^{\prime} / \mathcal{K}_{f}^{\prime \prime}$. One can identify $\mathcal{K}_{f}^{\text {phys }}$ with a dense subspace of $\mathcal{K}^{\text {phys }}$.

Theorem 2 (Observable fields for $d=26$ )

1. Let $F \in \mathcal{S}\left(\mathbb{R}^{d}, \mathcal{F}_{+}\left(\ell^{2}\right)\right)$ be a constrained test function. Then $\Phi(F)$ on $\mathcal{K}_{f}$ lifts to an operator $\Phi(F)$ on $\mathcal{K}_{f}^{\text {phys }}$ called an observable field

2. These satisfy $[\Phi(F), \Phi(G)]=-i<F, E G>$.

3. The representation $\mathcal{U}(a, \Lambda)$ on $\mathcal{K}$ lifts to a unitary representation $\mathcal{U}(a, \Lambda)$ on $\mathcal{K}^{\text {phys }}$ and

$$
\mathcal{U}(a, \Lambda) \Phi(F) \mathcal{U}(a, \Lambda)^{-1}=\Phi\left(F_{a, \Lambda}\right)
$$

Proof. Since $\Pi F \in \mathcal{H}_{+}^{\prime}$ we have that $\Phi(F)$ preserves $\mathcal{K}_{f}^{\prime}$. It also preserves $\mathcal{K}_{f}^{\prime \prime}$ since if $\Psi \in \mathcal{K}_{f}^{\prime \prime}$ and $\Xi \in \mathcal{K}_{f}^{\prime}$ then $<\Xi, \Phi(F) \Psi>=<\Phi(F) \Xi, \Psi>=0$. Since $\mathcal{K}_{f}^{\prime}$ is dense in $\mathcal{K}^{\prime}$ we have $<\Xi, \Phi(F) \Psi>=0$ for all $\Xi \in \mathcal{K}^{\prime}$ and hence $\Phi(F) \Psi \in \mathcal{K}_{f}^{\prime \prime}$. Hence $\Phi(F)$ acts on $\mathcal{K}_{f}^{\text {phys }}$.

The commutator follows from the commutator on $\mathcal{K}_{f}$

For the covariance first note that $F$ is constrained if and only if $F_{a, \Lambda}$ is constrained. This follows from the identity $\Pi F_{a, \Lambda}=U(a, \Lambda) \Pi F$ and the fact that $U(a, \Lambda)$ preserves $\mathcal{H}_{+}^{\prime}$. The operator $\mathcal{U}(a, \Lambda)$ preserves $\mathcal{K}_{f}^{\prime}$ since $U(a, \Lambda)$ preserves $\mathcal{H}_{+}^{\prime}$. We argue as before that it also preserves $\mathcal{K}_{f}^{\prime \prime}$ and so it lifts. The unitarity follows since it is inner product preserving, and the identity lifts from the identity on $\mathcal{K}_{f}$.

Remark. According to this theorem the observable fields have a local commutator. But can the fields themselves be localized? That is, are there constrained test functions $F \in \mathcal{C}_{0}^{\infty}\left(\mathbb{R}^{d}, \mathcal{F}_{+}\left(\ell^{2}\right)\right)$ ? Or is there some other way to get strictly localized operators? These are open questions. Without strictly localized fields we cannot get a vanishing result like Corollary [ ]. The best we can do is the following approximate result. If $F, G$ are constrained then it estimates the commutator for observable fields on $\mathcal{K}^{\text {phys }}$. Otherwise it refers to fields on $\mathcal{K}$.

Corollary 2 let $F, G \in \mathcal{S}\left(\mathbb{R}^{d}, \mathcal{F}_{+}\left(\ell^{2}\right)\right)$, and let a be in the spacelike region $\left|a^{0}\right|<$ $(1-\epsilon)|\vec{a}|$. Then as $|a| \rightarrow \infty$, we have for any $n>0$

$$
\left[\Phi\left(F_{a}\right), \Phi(G)\right]=-i<F_{a}, E G>=\mathcal{O}\left(|a|^{-n}\right)
$$


Proof. Since $E G$ is bounded we have

$$
\begin{aligned}
\left|<F_{a}, E G>\right| & \leq \mathcal{O}(1) \int_{\operatorname{supp}(E G)}\|F(x-a)\| d x \\
& \leq \mathcal{O}(1) \int_{\operatorname{supp}(E G)}(1+|x-a|)^{-n-d-1} d x \\
& \leq \mathcal{O}(1) d(a, \operatorname{supp}(E G))^{-n} \\
& \leq \mathcal{O}\left(|a|^{-n}\right)
\end{aligned}
$$

In the last step we use the fact that $\operatorname{supp}(E G)$ is contained in a set of the form $\{x \in$ $\left.\mathbb{R}^{d}:|\vec{x}| \leq x^{0}+C\right\}$. We omit the details. 


\section{References}

[1] J. Bognar, Indefinite Inner Product Spaces, Springer-Verlag (1974).

[2] R.C. Brower, Spectrum-generating algebra and no-ghost theorem for for the dual model, Phys. Rev. D6, (1972), 1655.

[3] J. Dimock, Algebras of local observables on a manifold, Commun. Math. Phys. 77, (1980), 219-228.

[4] J. Dimock, Quantized electromagnetic field on a manifold, Rev. Math. Phys. 4, (1992), 223-233.

[5] J. Dimock, Locality in Free String Field Theory, J. Math. Phys. 41, (2000), 40-61.

[6] I.B. Frenkel, H. Garland, G. J. Zuckerman, Semi-infinite cohomology and string theory, Proc. Nat. Acad. Sci. 83,(1986) 8442-8446.

[7] J. Glimm, A. Jaffe, Quantum field theory models, in Statistical Mechanics and Quantum Field Theory, C. DeWitt, R. Stora, eds., Gordon and Breach, New York, (1971).

[8] P. Goddard, C. Thorn, Compatibility of the dual pomeron with unitarity and the absence of ghosts in the dual resonance model, Phys. Lett. 40B, (1972), 235.

[9] M. Green, J. Schwartz, E. Witten, Superstring Theory, Cambridge University Press (1987).

[10] H. Grundling, C.A. Hurst, The operator quantization of the open bosonic string field algebra, Commun. Math. Phys. (1993), 473-525.

[11] R. Haag, Local Quantum Physics, Springer-Verlag (1992).

[12] H. Hata, H. Oda, Causality in covariant string field theory, Phys. Lett. B 394, (1997), 307-314.

[13] E. Hille and R.S. Phillips, Functional analysis and semi-groups, American Mathematical Society, Providence, (1957).

[14] C. Isham, Quantum field theory in curved spacetimes: a general mathematical framework, in Differential Geometrical Methods in Mathematical Physics II, K. Bleuler, H. Petry, A. Reetz, eds, Springer-Verlag (1978).

[15] D. Lowe, Causal properties of free string field theory, Phys. Lett. B326 (1994), 223-230.

[16] E. Martinec, The light cone in string theory, Class. Quant. Grav. 10 (1993) L187L192. 
[17] O. Nielsen, Direct integral theory, Marcel Dekker, New York, (1980).

[18] M.Reed, B.Simon, Methods of Modern Mathematical Physics II, Academic Press, New York, (1975).

[19] F. Strocchi, A. Wightman, Proof of the charge superselection rule in local relativistic quantum field theory, J. of Math. Phys. 15, (1974), 2198-2224.

[20] C. Thorn, String field theory, Phys. Rep. 175, (1989), 1-101.

[21] F. Warner, Foundations of Differentiable Manifolds and Lie Groups, SpringerVerlag (1983).

[22] S. Weinberg, The Quantum Theory of Fields I, Cambridge University Press (1995). 